Received: 2014.07.26 Accepted: 2014.09.16 Published: 2014.12 .23

Authors' Contribution: Study Design A Data Collection B Statistical Analysis C Data Interpretation D Manuscript Preparation E Literature Search F Funds Collection G

\section{The Impact of Treated Bacterial Infections within One Month before Living Donor Liver Transplantation in Adults}

\author{
ABCDEF Takanobu Hara \\ ACD Akihiko Soyama \\ AD Mitsuhisa Takatsuki \\ AD Masaaki Hidaka \\ AC Izumi Carpenter \\ AC Ayaka Kinoshita \\ D Tomohiko Adachi \\ D Amane Kitasato \\ D Tamotsu Kuroki \\ ACD Susumu Eguchi
}

Department of Surgery, Nagasaki University Graduate School of Biomedical Sciences, Nagasaki, Japan
Corresponding Author: Source of support:
Susumu Eguchi, e-mail: sueguchi@nagasaki-u.ac.jp

Departmental sources

Background: The impact of treated preoperative bacterial infections on the outcome of living-donor liver transplantation (LDLT) is not well defined. The aim of this study was to determine the frequency of pre-transplant bacterial infections within one month before LDLT and their impact on the post-transplant morbidity and mortality.

Material/Methods: We retrospectively reviewed the records of 50 adult LDLT recipients between January 2009 and October 2011. Patients were divided into two groups based on whether they had episodes of bacterial infections within one month before LDLT.

Results: There were 20 patients who required antimicrobial therapy for pre-transplant infections. The pre-transplant infections comprised urinary tract infections (35\%), cholangitis (10\%), pneumonia (10\%), bacteremia (5\%), spontaneous bacterial peritonitis (5\%), acute sinusitis (5\%), subcutaneous abscess (5\%), and empirical treatment (25\%). Patients with pre-transplant infections had higher Child-Pugh scores [median, 11 vs. 9.5, $\mathrm{P}<0.05$ ] and model for end-stage liver disease scores [median, 17.5 vs. $14, \mathrm{P}<0.05$ ] compared with the other patients. There were no correlations between the pathogens involved in the pre-transplant infections and those involved in post-transplant infections. The incidence of post-transplant infections was higher in the pre-transplant infection group within one week after LDLT, but was almost the same within one month after LDLT. The one-year survival rates were not significantly different between the groups.

Conclusions: Although pre-transplant infections are associated with a high risk of postoperative bacterial infection shortly after LDLT, they did not affect the short-term outcome when they had been appropriately treated before transplantation.

MeSH Keywords: Bacterial Infections • Liver Transplantation • Living Donors • Perioperative Care

Full-text PDF: http://www.annalsoftransplantation.com/abstract/index/idArt/892095

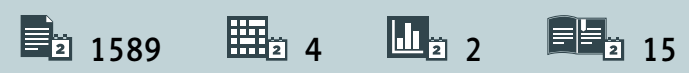




\section{Background}

Infection presents a higher risk when one considers the immunosuppression required by patients after transplantation. Therefore, patients with an active uncontrolled infection cannot undergo transplantation. Since deceased donor liver transplantation (DDLT) is usually performed as an emergent surgery, a preceding preoperative evaluation for occult infection cannot always be extensively performed. Despite this drawback of emergency surgery, some studies have revealed that histories of pre-transplant infections do not affect the outcomes of DDLT [1,2]. However, the outcomes of patients with pre-transplant infections have not been clarified in living donor liver transplantation (LDLT). Because of the shortage of deceased donors, LDLT has become an important therapeutic option for patients with end-stage liver disease. Since LDLT is generally performed as an elective surgery, it is possible to optimize the timing of transplantation depending on the recipient condition [3]. Patients and medical staff can make adequate preparations, including treatment for occult infections, before the operation. However, despite cautious preparation, patients may still develop pre-transplant infections, because patients with liver cirrhosis have increased susceptibility to bacterial infections and a risk of sepsis $[1,4]$.

We usually perform LDLT as scheduled when the patients' pre-transplant infections have been cured with documentation of eradication before the operation. However, if pretransplant infections affect the patient outcome in LDLT, it would be necessary to reconsider and postpone the operation. Since postponement of LDLT is often possible, clarifying the impact of pre-transplant infections on the post-LDLT outcomes is important.

The objectives of this study were to examine the details of the perioperative infections in LDLT recipients and to assess whether pre-transplant infections affect the early post-transplant outcome.

\section{Material and Methods}

We retrospectively analyzed 50 adult patients ( 27 males) who had undergone initial LDLT at Nagasaki University Hospital from January 2009 to September 2011. All transplantations were approved by the ethics committee of Nagasaki University Hospital.

\section{Diagnosis of infections}

Bacterial infections occurring within one month before LDLT were defined as pre-transplant infections in this study. Pre- and post-transplant infections were defined according to the criteria proposed by the Centers for Disease Control and Prevention [5]

\section{Immunosuppression therapy}

The standard immunosuppression regimen comprised tacrolimus and steroids. The trough level of tacrolimus was adjusted to $10-15 \mathrm{ng} / \mathrm{ml}$ until one month after surgery, and was tapered $10 \mathrm{ng} / \mathrm{ml}$ or less thereafter. Methyl prednisolone was administered at $1 \mathrm{~g}$ intravenously (i.v.) just before reperfusion during surgery. During the postoperative period, we administered methyl prednisolone at a dose of $0.5 \mathrm{mg} / \mathrm{kg}$ i.v. four times a day for the first three postoperative days, followed by $0.5 \mathrm{mg} / \mathrm{kg}$ twice a day for the next three days. Thereafter, the i.v. steroid was switched to oral prednisolone at $0.5 \mathrm{mg} / \mathrm{kg}$ once a day at seven days after transplantation, and the steroid was discontinued by three months after LDLT. Mycophenolate mofetil was added for ABO-incompatible LDLT cases and patients who were intentionally kept at lower trough levels of tacrolimus due to renal dysfunction.

\section{Antimicrobial therapy}

Antimicrobial prophylaxis comprised cefazolin ( $4 \mathrm{~g} /$ day) and ampicillin (4 g/day). These medications were started $30 \mathrm{~min}$ utes before laparotomy, and continued to be administered for 48 hours after the operation. The prophylaxis regimen was used for patients without pre-transplant infections and patients with pre-transplant infections who had completed treatment at the time of the operation.

\section{Statistical analysis}

The IBM SPSS Statistics 20 software program was used for the statistical analyses. The Mann-Whitney $U$ test was used to analyze continuous data, and the chi-square test was used for categorical data. The overall survival was calculated with the Kaplan-Meier method, and data were compared with the log-rank test. A multivariate analysis using a Cox proportional hazards model was used to assess the factors predicting the survival rate one year after LDLT. We considered a value of $P<0.05$ to be statistically significant.

\section{Results}

\section{The characteristics of patients}

The indications for liver transplantation were liver cirrhosis due to hepatitis virus infection $(n=34,68 \%)$, primary biliary cirrhosis $(n=4,8 \%)$, primary sclerosing cholangitis $(n=3$, $6 \%)$, alcoholic liver cirrhosis $(n=3,6 \%)$, fulminant hepatic failure $(n=2,4 \%)$, and other diseases $(n=4,8 \%)$. Twenty patients (40\%) had pre-transplant infections. Patients with pre-transplant infections had higher model for end-stage liver disease (MELD) scores (median=17.5 vs. 14, $\mathrm{P}<0.05$ ) and Child-Pugh 
Table 1. Characteristics of patients.

\begin{tabular}{|c|c|c|c|c|c|}
\hline \multirow[b]{3}{*}{ Age } & \multicolumn{4}{|c|}{ Pretransplant infections } & \multirow{3}{*}{$\begin{array}{r}\text { P Value } \\
0.789\end{array}$} \\
\hline & \multicolumn{2}{|c|}{$(+) n=20$} & \multicolumn{2}{|c|}{$(-) n=30$} & \\
\hline & 55 & $(30-72)$ & 55 & $(27-72)$ & \\
\hline Gender, male & 7 & $(35 \%)$ & 20 & $(66 \%)$ & 0.028 \\
\hline MELD score & 17.5 & $(9-43)$ & 14 & $(7-27)$ & 0.028 \\
\hline Child-Pugh score & 11 & $(6-15)$ & 9.5 & $(5-13)$ & 0.018 \\
\hline Hepatitis virus infection & 8 & $(40 \%)$ & 26 & $(87 \%)$ & $<0.01$ \\
\hline Cholestatic liver disease & 6 & $(30 \%)$ & 1 & $(3.3 \%)$ & 0.012 \\
\hline $\mathrm{HCC}$ & 6 & $(30 \%)$ & 21 & $(70 \%)$ & $<0.01$ \\
\hline Operaion time (min) & 802.5 & $(598-1159)$ & 802 & $(654-1129)$ & 0.961 \\
\hline Blood loss $(\mathrm{ml})$ & 5700 & $120-17600)$ & 4150 & $(520-18400)$ & 0.075 \\
\hline Pretransplant dialysis & 4 & $(20 \%)$ & 1 & $(3 \%)$ & 0.076 \\
\hline Pretransplant ICU stay & 3 & $(15 \%)$ & 1 & $(3.3 \%)$ & 0.170 \\
\hline Left lobe graft & 17 & $(85 \%)$ & 20 & $(66 \%)$ & 0.131 \\
\hline Hepaticojejunostomy & 4 & $(20 \%)$ & 1 & $(3.3 \%)$ & 0.076 \\
\hline Incompatible ABO blood type & 2 & $(10 \%)$ & 4 & $(13.3 \%)$ & 0.544 \\
\hline Post LDLT ICU stay (days) & 5 & $(1-36)$ & 7 & $(2-20)$ & 0.259 \\
\hline Post LDLT hospital stay (days) & 47.5 & $(16-195)$ & 47.5 & $(17-140)$ & 0.513 \\
\hline
\end{tabular}

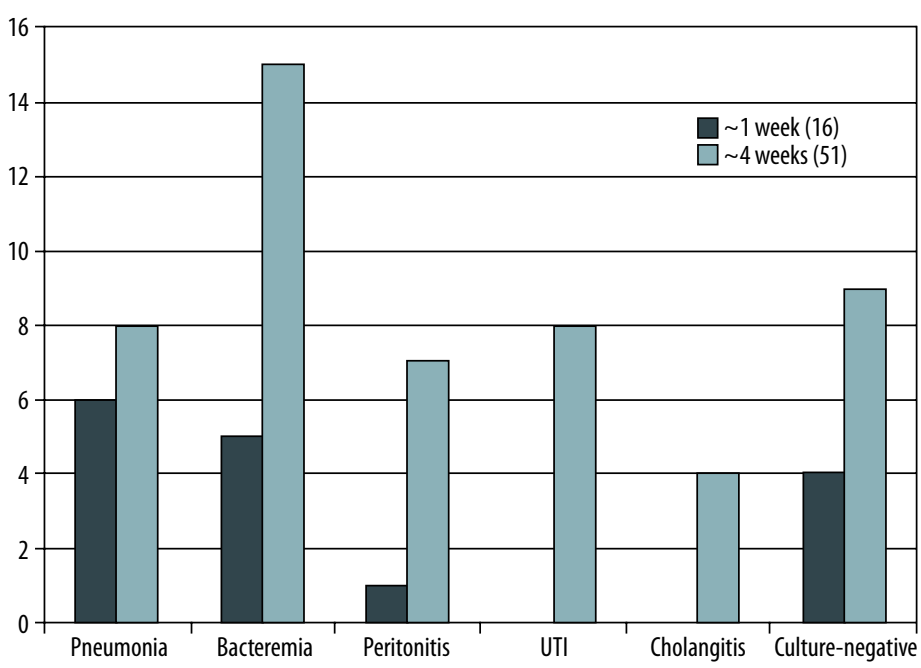

Figure 1. A comparison of the types of infections after LDLT. Pneumonia was the most common infection that occurred within one week after LDLT. UTI and cholangitis were not detected during this period. On the other hand, bacteremia was the most common infection one month after LDLT.

scores (median=11 vs. 9.5, $\mathrm{P}<0.05$ ) than patients without infections. The rates of hepatitis virus infections ( $40 \%$ vs. $87 \%$, $\mathrm{P}<0.01)$ and hepatocellular carcinoma ( $30 \%$ vs. $70 \%, \mathrm{P}<0.01)$ were lower in the pre-transplant infection group. These patients also had more cholestatic liver diseases, which included primary biliary cirrhosis and primary sclerosing cholangitis ( $30 \%$ vs. $3.3 \%, P=0.01$ ). There were no significant differences in other indications (Table 1).

\section{Pre-transplant infections}

The pre-transplant infections comprised urinary tract infections (UTI) ( $n=7,35 \%)$, cholangitis $(n=2,10 \%)$, pneumonia $(n=2,10 \%)$, bacteremia $(n=1,5 \%)$, spontaneous bacterial peritonitis (SBP) ( $n=1,5 \%)$, acute sinusitis $(n=1,5 \%)$, and subcutaneous abscess $(n=1,5 \%)$. The other five patients $(25 \%)$ did not completely match the criteria proposed by the Centers for 
Table 2. The details of post-transplant infections.

\begin{tabular}{|c|c|c|c|}
\hline Infection/Pathogens & 0-1 week & -1 month & $\mathbf{N}$ \\
\hline Bacteremia & 5 & 10 & 15 \\
\hline Pseudomonas aeruginosa & 2 & 4 & 6 \\
\hline Enterococcus faecium & 1 & 1 & 2 \\
\hline Acinetobacter baumannii & & 1 & 1 \\
\hline Bacteroides fragilis & 1 & & 1 \\
\hline Enterobacter cloacae & & 1 & 1 \\
\hline Escherichia coli & & 1 & 1 \\
\hline Klebsiella pneumoniae & & 1 & 1 \\
\hline MRCNS & 1 & & 1 \\
\hline Staphylococcus epidermidis & & 1 & 1 \\
\hline Pneumonia & 6 & 2 & 8 \\
\hline Staphylococcus epidermidis & 2 & & 2 \\
\hline Enterobacter cloacae & 1 & & 1 \\
\hline Klebsiella pneumoniae & 1 & & 1 \\
\hline MRSA & 1 & 1 & 2 \\
\hline Pseudomonas aeruginosa & 1 & 1 & 2 \\
\hline UTI & 0 & 8 & 8 \\
\hline Enterococcus faecium & & 3 & 3 \\
\hline Klebsiella pneumoniae & & 2 & 2 \\
\hline Citrobacter freundii & & 1 & 1 \\
\hline MRCNS & & 1 & 1 \\
\hline Staphylococcus epidermidis & & 1 & 1 \\
\hline Peritonitis & 1 & 6 & 7 \\
\hline Enterococcus faecium & 1 & & 1 \\
\hline Enterococcus faecalis & & 2 & 2 \\
\hline Pseudomonas aeruginosa & & 2 & 2 \\
\hline Enterococcus raffinosus & & 1 & 1 \\
\hline Staphylococcus epidermidis & & 1 & 1 \\
\hline Cholangitis & 0 & 4 & 4 \\
\hline Enterococcus faecium & & 4 & 4 \\
\hline Culture-negative & 4 & 5 & 9 \\
\hline
\end{tabular}

MRCNS - methicillin-resistant coagulase negative staphylococci; UTI - urinary tract infection. 
Table 3. Incidence of post-transplant infections.

\begin{tabular}{lccccc} 
& \multicolumn{1}{c}{ Pre-transplant infections } & P Value \\
\hline Within 1 week after LDLT & $(+) \mathrm{N}=20$ & $(-) \mathrm{N}=30$ & 0.028 \\
\hline Within 2 weeks after LDLT & $10 \quad(50 \%)$ & 6 & $(20 \%)$ \\
\hline Within 1 month after LDLT & 15 & $(75 \%)$ & 16 & $(53 \%)$ & 0.105 \\
\hline
\end{tabular}

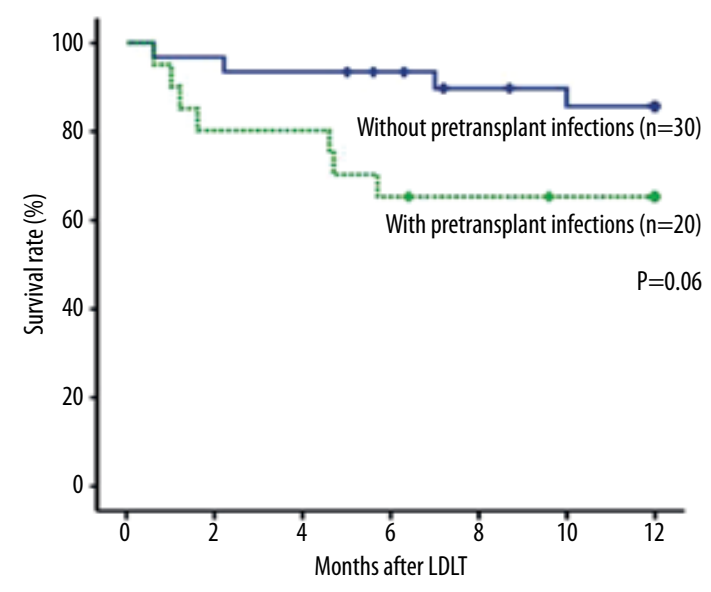

Figure 2. The survival rates of patients with and without pretransplant infections. The one-year survival rate was lower in the pre-transplant infection group, but the difference was not significant ( $86 \%$ vs. $65 \%, P=0.06$ ).

Disease Control and Prevention [5]. However, all five patients were diagnosed to have bacterial infections based on their clinical status (fever, chills and elevated levels of inflammatory parameters), and thus were treated empirically. The clinical conditions of all patients improved rapidly after the administration of antimicrobial therapy. No patients had combined episodes of infection. Pathogens were detected in 11 of 20 patients (12 pathogens). Escherichia coli was the most common pathogen in the pre-transplant period $(n=6)$, followed by
Enterococcus faecium $(\mathrm{n}=2)$, Enterococcus avium $(\mathrm{n}=1)$, Proteus mirabilis $(n=1)$, Streptococcus epidermidis $(n=1)$, and Methicillinresistant Staphylococcus aureus $(\mathrm{n}=1)$.

\section{Post-transplant infections}

Of the 50 patients, 16 patients (16 episodes) developed posttransplant infections within one week after LDLT. Pneumonia was the most common infection during this period. On the other hand, 36 of the 50 patients (51 episodes) had post-transplant infections within one month after LDLT. Bacteremia was the most common infection during this period (Figure 1). The details of the causative bacterial pathogens after LDLT are shown in Table 2. Among the patients with pre-transplant infections, the previous pathogens were not found after LDLT in any of the patients. The post-transplant infection rate was significantly higher in the pre-transplant infection group during the first week after LDLT ( $50 \%$ vs. $20 \%, P<0.05)$, but was not significantly different within one month after LDLT (85\% vs. 63\%) (Table 3).

\section{Patient outcomes}

The one-year survival rate tended to be lower in the pre-transplant infection group ( $65 \%$ vs. $86 \%, \mathrm{P}=0.06$; Figure 2$)$. A multivariate analysis showed that a higher MELD score $(P<0.05)$ was a significant risk factor for a decreased one-year survival after LDLT (Table 4). A pre-transplant infection did not significantly adversely affect the one-year survival after adjusting for other factors.

Table 4. Multivariate analysis for 1 year survival.

\begin{tabular}{|c|c|c|c|}
\hline Variable & \multicolumn{2}{|c|}{ HR ( $95 \%$ confidence interval) } & P Value \\
\hline Age $(>60)$ & 3.77 & $(0.34-41.67)$ & 0.279 \\
\hline MELD $(>20)$ & 28.38 & $(1.40-576.45)$ & 0.029 \\
\hline Child-Pugh (>9) & 1.81 & $(0.21-15.57)$ & 0.587 \\
\hline Hepatitis virus infection & 1.62 & $(0.23-11.61)$ & 0.633 \\
\hline $\mathrm{HCC}$ & 6.91 & $(0.64-74.90)$ & 0.112 \\
\hline Incompatible $\mathrm{ABO}$ blood type & 0.17 & $(0.010-2.77)$ & 0.210 \\
\hline Pretransplant infections & 2.77 & $(0.43-18.02)$ & 0.286 \\
\hline
\end{tabular}




\section{Discussion}

In the study, we found that the presence of bacterial infections prior to LDLT was not a risk factor for bacterial infections developing during the first month after LDLT. The one-year survival rate was lower in the pre-transplant infection group, but the rates were not significantly different. We thought that the tendency might have been based on the differences in the background of the patients, because those with pre-transplant infections had higher Child-Pugh scores and MELD scores. In fact, the MELD score was the only significant risk factor for the one-year survival in this study.

Surgical outcomes are largely influenced by the pre-surgical conditions [6], and the MELD score has a crucial role in predicting early postoperative mortality after DDLT [3,7]. The same result was also reported in LDLT [8,9]. However, other studies have concluded that the MELD score had no correlation with graft or patient survival [10-12]. We found that a MELD score $>20$ was associated with a lower one-year survival rate.

The studies concerning the effects of pre-transplant bacterial infections have been limited. One study showed that patients with an episode of pre-transplant SBP had a higher incidence of post-transplant complications, infections, and early transplant mortality [13]. On the other hand, three studies concluded that a pre-transplant SBP history did not affect the post-transplant outcome $[2,14,15]$. Sun et al. divided their 100 DDLT cases into two groups; a pre-transplant infection group (32/100) and a non-infection group (68/100). They concluded that pre-transplant infections were not a significant risk factor for poor outcomes if the post-transplant infections were adequately treated [1]. Our study evaluated the influence of preoperative infections

\section{References:}

1. Sun HY, Cacciarelli TV, Singh N: Impact of pretransplant infections on clinical outcomes of liver transplant recipients. Liver Transpl, 2010; 16: 222-28

2. Mouzer R, Malik SM, Nasr J et al: Spontaneous bacterial peritonitis before liver transplantation does not affect patient survival. Clin Gastroenterol Hepatol, 2010; 8: 623-28

3. Saab S, Wang V, Ibrahim AB et al: MELD score predicts 1-year patient survival post-orthotopic liver transplantation. Liver Transpl, 2003; 9: 473-76

4. Foreman MG, Mannino DM, Moss M: Cirrhosis as a risk factor for sepsis and death: analysis of the National Hospital Discharge Survey. Chest, 2003; 124: $1016-20$

5. Garner JS, Jarvis WR, Emori TG et al: CDC definitions for nosocomial infections, 1988. Am J Infect Control, 1988; 16: 128-40

6. Clavien PA, Barkun J, de Oliveira ML et al: The Clavien-Dindo classification of surgical complications: five-year experience. Ann Surg, 2009; 250: 187-96

7. Habib S, Berk B, Chang CC et al: MELD and prediction of post-liver transplantation survival. Liver Transpl, 2006; 12: 440-47

8. Marubashi S, Dono K, Asaoka T et al: Risk factors for graft dysfunction after adult-to-adult living donor liver transplantation. Transplant Proc, 2006; 38: $1407-10$ in LDLT cases for the first time. Despite the difference in their backgrounds, our results were almost the same as the previous reports which examined DDLT patients. Interestingly, patients with pre-transplant infections had more infectious episodes within one week after LDLT. We could not determine the reason for this phenomenon, but it might reflect the poorer general condition of the patients at the time of transplantation. On the other hand, since no patient exhibited a recurrent infection with the pre-transplant pathogens in the early post-operative period, we considered that the pre-transplant infections were successfully treated and the post-transplant infections were all new. This result suggested that the treatment of pre-transplant pathogens did not need to be continued in the post-transplant period. To improve the rate of early detection of post-transplant infections, patients should be recognized as a group at high risk of bacterial infections. The duration of antimicrobial prophylaxis, timing of immunosuppressant therapy, and the loading doses should be intensively discussed considering the patient's condition.

\section{Conclusions}

Pre-transplant infections did not affect the incidence of posttransplant infections within one month after LDLT, and did not affect the one-year survival rate. However, pre-transplant infections were associated with a high risk of post-transplant infection within the first week after LDLT.

\section{Statement}

The authors declare no conflict of interest. This work was not funded by any educational or commercial organization.

9. Kaido T, Egawa $\mathrm{H}$, Tsuji $\mathrm{H}$ et al: In-hospital mortality in adult recipients of living donor liver transplantation: experience of 576 consecutive cases at a single center. Liver Transpl, 2009; 15: 1420-25

10. Hayashi PH, Forman L, Steinberg T et al: Model for End-Stage Liver Disease score does not predict patient or graft survival in living donor liver transplant recipients. Liver Transpl, 2003; 9: 737-40

11. Yi NJ, Suh KS, Lee HW et al. Improved outcome of adult recipients with a high model for end-stage liver disease score and a small-for-size graft. Liver Transpl, 2009; 15: 496-503

12. Wai CT, Woon WA, Tan YM et al: Pretransplant Model for End-stage Live Disease score has no impact on posttransplant survival in living donor liver transplantation. Transplant Proc, 2012; 44: 396-98

13. Ukah FO, Merhav H, Kramer D et al: Early outcome of liver transplantation in patients with a history of spontaneous bacterial peritonitis. Transplant Proc, 1993; 25: 1113-15

14. Altman C, Grangé JD, Amiot $X$ et al: Survival after a first episode of spontaneous bacterial peritonitis. Prognosis of potential candidates for orthotopic liver transplantation. J Gastroenterol Hepatol, 1995; 10: 47-50

15. Van Thiel DH, Hassanein T, Gurakar A et al: Liver transplantation after an acute episode of spontaneous bacterial peritonitis. Hepatogastroenterology, 1996; 43: 1584-88 